\section{Ciencia y tecnología en la provincia de Buenos Aires}

Resumen: La provincia de Buenos Aires (PBA) reúne poco más del 30\% de la inversión pública y privada total en I+D de Argentina. Su magnitud es tal que, si fuese un país, podría ocupar el cuarto lugar en inversión en este rubro, después de Brasil, México y Argentina. Es el distrito que produce la mayor cantidad de publicaciones científicas y el que tiene mayor número de personas dedicadas a actividades de I+D. Sin embargo, no ha logrado organizar toda esta potencialidad, ni articularla a su desarrollo productivo y social. El presente artículo busca poner de relieve la importancia y necesidad de contar con una política provincial de CyT que responda a las demandas socioproductivas de la PBA. Para ello se muestra, por un lado, las capacidades CyT con las que cuenta. Por otro lado, se presenta un conjunto de propuestas para que el complejo de instituciones CyT que trabajan en el territorio de la PBA pueda colaborar en la resolución de los graves problemas que afectan a la región.

Palabras claves: CyT en la Provincia de Buenos Aires, Comisión de Investigaciones Científicas, polo CyT, plan estratégico provincial de CyT, tecnología para la producción popular.

Science and Technology in the Province of Buenos Aires Capabilities and Proposals

Abstract: The Province of Buenos Aires (PBA) gathers just over 30\% of total public and private investment in $R \& D$ in Argentina. Its magnitude is such that, if it were a country, it could occupy the fourth place in investment in this area, after Brazil, Mexico and Argentina. It is the district that produces the most scientific publications and the one with the largest number of people dedicated to R\&D activities. However, it has not managed to organize all this potential, nor to articulate it to its productive and social development. This article seeks to highlight the importance and need of having a provincial S\&T policy that responds to the socio-productive demands of the PBA. For this, it shows, on the one hand, the S\&T capabilities that it has. On the other hand, a set of proposals is presented so that the complex of S\&T institutions working in the PBA territory can collaborate in solving the serious problems that affect the region.

Keywords: S\&T in the Province of Buenos Aires, Scientific Research Commission, S\&T pole, S\&T provincial strategic plan, technology for popular production.

\section{Ciência e Tecnologia na Província de Buenos Aires Capacidades e propostas}

Resumo: A Província de Buenos Aires (PBA) reúne pouco mais de 30\% do investimento público e privado total em $P \& D$ na Argentina. Sua magnitude é tal que, se fosse um país, poderia ocupar o quarto lugar em investimentos nessa área, depois do Brasil, México e Argentina. É o distrito que produz mais publicações científicas e aquele com o maior número de pessoas dedicadas às atividades de P\&D. No entanto, não conseguiu organizar todo esse potencial nem articulá-lo ao seu desenvolvimento produtivo e social. Este artigo procura destacar a importância e a necessidade de uma política provincial de C\&T que responda às demandas sócio-produtivas do PBA. Para isso, mostra, primeiramente, os recursos de C\&T que possui. Em segundo lugar, é apresentado um conjunto de propostas para que o complexo de instituições de C\&T, que trabalham no território do PBA, possa colaborar na solução dos graves problemas que afetam a região. Palavras-chave: C\&T na província de Buenos Aires, Comissão de Pesquisa Científica, pólo de C\&T, plano estratégico provincial de C\&T, tecnologia para produção popular.

\section{Ciencia Tecnología y Política \\ Año 3 N 4 Mayo 2020}

*Artículo elaborado colectivamente en el marco de la Cátedra Libre CPS por Gabriel Bilmes, Santiago

Liaudat, Ignacio Ranea Sandoval, Julián Bilmes y Gabriel Baum.

Con aportes de Marcela Fushimi y Leandro Andrin

Año $3 \mathrm{~N}^{\circ} 4$ Mayo 2020 Fecha de recibido: 03/03/2020 Fecha de aprobado: 30/04/2020 https://doi.org/10.24215/26183188e034 https://revistas.unlp.edu.ar/CTyP ISSN 2618-3188

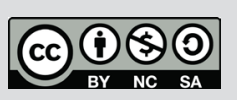

Esta obra está bajo licencia Creative Commons Atribución-NoComercial-Compartirlgual 4.0 Internacional http://creativecommons.org/licenses/bync-sa/4.O/deed.es_AR 


\section{Ciencia y tecnología en la provincia de Buenos Aires. Capacidades y propuestas}

*Artículo elaborado colectivamente en el marco de la Cátedra Libre CPS por Gabriel Bilmes, Santiago Liaudat, Ignacio Ranea Sandoval, Julián Bilmes y Gabriel Baum. Con aportes de Marcela Fushimi y Leandro Andrini

Resumen: La provincia de Buenos Aires (PBA) reúne poco más del 30\% de la inversión pública y privada total en I+D de Argentina. Su magnitud es tal que, si fuese un país, podría ocupar el cuarto lugar en inversión en este rubro, después de Brasil, México y Argentina. Es el distrito que produce la mayor cantidad de publicaciones científicas y el que tiene mayor número de personas dedicadas a actividades de I+D. Sin embargo, no ha logrado organizar toda esta potencialidad, ni articularla a su desarrollo productivo y social. El presente artículo busca poner de relieve la importancia y necesidad de contar con una política provincial de CyT que responda a las demandas socioproductivas de la PBA. Para ello se muestra, por un lado, las capacidades CyT con las que cuenta. Por otro lado, se presenta un conjunto de propuestas para que el complejo de instituciones CyT que trabajan en el territorio de la PBA pueda colaborar en la resolución de los graves problemas que afectan a la región.

\section{Introducción}

Es difícil dimensionar la importancia que la Provincia de Buenos Aires (PBA) tiene en la estructura socioeconómica argentina. Algunos datos sirven para graficarlo. Casi el 40\% de la población nacional es bonaerense y más de un tercio del Producto Bruto Interno es aportado por actividades económicas desarrolladas en la provincia. Entre ellas se destaca un peso preponderante de la actividad industrial, concentrando el mayor número de establecimientos del país, y de la actividad agrícola-ganadera (un $37 \%$ del valor agregado del sector agropecuario nacional). ${ }^{1}$ Se trata de un altísimo grado de concentración demográfica y productiva con enormes consecuencias en la historia argentina, tanto a nivel político como económico y cultural.

Esta centralidad y concentración se refleja también en el sector de ciencia y tecnología (CyT). La PBA reúne poco más del 30\% de la inversión pública y

${ }^{1}$ Elaboración propia a partir de datos obtenidos de la página web del Instituto Nacional de Estadísticas y Censos (INDEC). 
| Ciencia, Tecnología y Política | Año 3 | №4 | Mayo 2020 | ISSN 2618-3188 | www.revistas.un|p.edu.ar/CTyP |

privada total en investigación y desarrollo (I+D) del país, seguida de cerca por la Ciudad Autónoma de Buenos Aires (CABA) y más lejos por las provincias de Córdoba y Santa Fe (alrededor de un $8 \%$ y 5\% respectivamente). Las otras veinte provincias argentinas reúnen tan sólo el 25\% restante. ${ }^{2}$ Su magnitud es tal que, si fuese un país, ocuparía el cuarto lugar en inversión en I+D después de Brasil, México y Argentina, disputando ese lugar con Colombia, Chile y Venezuela. ${ }^{3}$ El territorio bonaerense, además, es el distrito que encabeza la producción nacional en CyT medida en cantidad de publicaciones científicas. En cuanto a recursos humanos, la PBA es el distrito con mayor número de personas dedicado a actividades de I+D del país calculado en EJC (Equivalente Jornada Completa), seguido de cerca por la CABA ( $28 \%$ y $25 \%$ del total nacional, respectivamente). ${ }^{4}$

Sin embargo, la provincia no ha logrado organizar toda esta potencialidad, ni articularla a su desarrollo productivo y social. A la ausencia de una política provincial de CyT, se suma una muy baja complejidad tecnológico-organizacional. El resultado es una interconexión muy débil entre instituciones de CyT y sector productivo, especialmente Pequeñas y Medianas Empresas (PyMEs), que ha sido una característica incluso en años de bonanza económica y en los que el gobierno nacional apoyó fuertemente al sector científico-tecnológico (2003-2015). Es de- cir, no se trata sólo de un problema coyuntural, sino estructural. Entre las causas que contribuyen a esta situación se podría enumerar la concentración económica en manos de empresas extranjeras que realizan las inversiones de I+D fuera del país, y actores sociales y productivos que, por diversas razones, no vislumbran sus necesidades como demandas de conocimiento. Con el agravante de que ambos motivos se retroalimentan, favoreciendo un círculo vicioso que caracteriza a los países de la periferia global: la falta de articulación entre gobierno, actividad científico-tecnológica y estructura socioproductiva.

Por otro lado, si bien se trata de un problema estructural, la coyuntura de los años recientes ha agravado enormemente la situación. Las políticas neoliberales impulsadas por la gobernadora María Eugenia Vidal (2015-2019) -en sintonía con el gobierno nacional de Mauricio Macri- se caracterizaron por fomentar la especulación financiera, produciendo un alto grado de desindustrialización y recesión económica junto a un agravamiento del pasivo provincial. Como producto de estas políticas, nos encontramos con un Estado sumamente debilitado frente al enorme desafío de lidiar con la emergencia social y económica en curso. ${ }^{5}$

Sobre este trasfondo, el presente artículo se propone poner de relieve la importancia y necesidad de contar con una política provincial de ciencia y tecno-

\footnotetext{
2 Los valores son un promedio del período 2007-2017 en base a información provista en la página web de la Dirección Nacional de Información Científica (DNIC). La inversión en I+D realizada en el territorio bonaerense a valores constantes en pesos creció un $50 \%$ entre 2007 y 2015 (pasando de 927 a 1.526 millones de pesos), reflejando la política de fortalecimiento de la CyT y el crecimiento económico durante los gobiernos de Cristina Fernández de Kirchner. En cambio, en los primeros años del gobierno de Mauricio Macri la inversión en I+D en la provincia tuvo un fuerte descenso del orden del 27\%, alcanzando en 2017 una inversión de tan sólo 1.114 millones de pesos.

3 Promedio del período período 2007-2017, medido tanto en dólares corrientes como en dólares PPC (paridad de poder de compra). Estimación realizada en base a datos obtenidos de las páginas web de DNIC y de la Red de Indicadores en Ciencia y Tecnología Interamericana e Iberoamericana (RICYT). Se agradece la colaboración de Sergio Rodríguez y Érica Carrizo.

${ }^{4}$ La cantidad de investigadores y becarios por cada 100.000 habitantes es el único indicador agregado que no favorece a la PBA. Con un valor de 86, se ubica por debajo del promedio nacional (114), y muy lejos de la CABA (399) y Santa Fe (123). El volumen poblacional bonaerense lleva a la baja el guarismo provincial. Datos obtenidos de la página web de la DNIC referidos al año 2018.

${ }_{5}^{5}$ Durante el período 2015-2019 cerraron sus puertas en la provincia más de 5.500 PyMEs, mientras que la pobreza y la indigencia alcanzaron a 38 y a 8 de cada 100 bonaerenses, respectivamente (Basualdo, Manzanelli y Bona, 2019). En cuanto a la deuda pública, indicadores oficiales señalan un notorio incremento del monto, junto a un aumento del peso de la deuda en dólares, mayormente contraída con bonistas internacionales, y un desmejoramiento del perfil de vencimientos, perjudicando seriamente la sostenibilidad provincial (Ministerio de Hacienda y Finanzas, 2020).
} 
logía que responda a las demandas socioproductivas de la PBA. Para ello se muestra, en primer lugar, cuáles son las capacidades científico-tecnológicas con las que cuenta la provincia, tanto a nivel de infraestructura y organismos públicos de CyT como de recursos humanos. Se distingue entre aquellas capacidades que están instaladas en la provincia, pero que son parte del Estado Nacional o del sector empresarial, de aquellas que son dependientes directamente del Estado Provincial. En segundo lugar, se pone a consideración un conjunto de propuestas en la aspiración de que el complejo de instituciones CyT que trabajan en el territorio bonaerense, mayormente desarticuladas entre sí y respecto a la realidad provincial, pueda configurarse como un Sistema de Ciencia, Tecnología e Innovación que colabore en la resolución de los graves problemas que afectan a la región.

\section{Capacidades CyT instaladas en la provincia de Buenos Aires}

En Argentina, más del 65\% de la producción científica se realiza en las Universidades Públicas, y es la Provincia de Buenos Aires el distrito del país con mayor cantidad de estas instituciones. ${ }^{6}$ En su territorio se despliegan más de 20 universidades nacionales y 7 Facultades Regionales de la Universidad Tecnológica Nacional (UTN), además de la presencia de dependencias de universidades e institutos universitarios radicados en la Ciudad Autónoma de Buenos Aires. Estas instituciones contienen alrededor de un cuarto del total nacional de la población universitaria de grado, que ha crecido en forma notable con la creación entre 2000 y 2017 de un conjunto de nuevas universidades alojadas en el Conurbano Bonaerense (la PBA pasó de tener unos 300.000 estudiantes a inicios de los años 2000 a cerca de medio millón en 2017). El 90\% de estos estudiantes universitarios asiste a universidades públicas, superando la media nacional del $80 \%$ ?

En el nivel de posgrado, sumando especializaciones, maestrías y doctorados, la PBA reúne un $26 \%$ de los estudiantes del país, alcanzando los 40.365 alumnos/as en el año 2017. En el nivel de doctorado alcanza un 29\% del total nacional. Sin embargo, cabe destacar que existe dentro de la provincia también un alto grado de concentración. La Universidad Nacional de La Plata posee el $40 \%$ de los doctorandos de la provincia, seguida de lejos por la Universidad Nacional de Mar del Plata (13\%) y la Universidad Nacional del Sur (12\%). Por año se gradúan unos 500 doctores y unos 1.000 magísteres de universidades instaladas en la Provincia de Buenos Aires. La distribución disciplinar de las matrículas de doctorado muestra que el $28 \%$ corresponde a Ciencias Sociales (con primacía de Derecho), un 23\% a las Ciencias Básicas (más de la mitad en Ciencias Biológicas y Naturales) y un $22 \%$ a las Ciencias Aplicadas, concentradas en agronomía/agropecuarias, ingeniería, informática y tecnología. ${ }^{8}$

Por otra parte, se radican en la provincia cuatro Escuelas Técnicas y/o Tecnológicas de nivel secundario dependientes de las Universidades Nacionales de Quilmes, General Sarmiento y Avellaneda y de la Tecnológica Nacional (ubicada en Villa Ballester en la ex Escuela Fábrica de la empresa Siemens Argentina). Por último, aunque es colateral a la formación de recursos humanos, se destaca la presencia de la megamuestra de ciencia, tecnología e innovación Tecnópolis, ubicada en Villa Martelli (Vicente López), uno de los principales espacios de divulgación pública y comunicación de la CyT en nuestro país.

\footnotetext{
6 Según datos de la base SCOPUS para los años 2010 y 2015. Fuente: OCTS, 2018.

7 Elaboración propia a partir de datos obtenidos de la SPU (2019) referidos al año 2017.

${ }^{8}$ Elaboración propia a partir de datos obtenidos de la SPU (2019) referidos al año 2017.
} 
Junto a las Universidades Nacionales, el Consejo Nacional de Investigaciones Científicas y Técnicas (CONICET) es el organismo nacional con más presencia en la Provincia de Buenos Aires. Con 3.091 investigadores/as, 2.895 becarios/as y 294 Unidades Ejecutoras, la PBA es el distrito con más cantidad de personal del CONICET (alrededor del 27\%), seguido de cerca por la Ciudad Autónoma de Buenos Aires. ${ }^{9}$

Por otra parte, localizados en territorio bonaerense se encuentran algunos de los organismos nacionales descentralizados de CyT más importantes del país, ya sea sus sedes centrales, o bien algunas dependencias, unidades de experimentación, observación o extensión. A saber:

- Instituto Nacional de Tecnología Industrial (INTI). Tiene sede central en Miguelete (San Martín) y unidades de extensión en Tandil y Lobos;

- Instituto Nacional de Tecnología Agropecuaria (INTA). Cuenta con Centros Regionales, Estaciones Experimentales, Centros de Investigación y Unidades de Extensión en diferentes puntos de la provincia;

- Instituto Nacional de Investigación y Desarrollo Pesquero (INIDEP). Ubicado en la ciudad de Mar del Plata;

- Instituto Nacional del Agua (INA). Localizado en el Partido de Ezeiza;

- Comisión Nacional de Energía Atómica (CNEA). Cuenta con el Centro Atómico Constituyentes (San Martín) y el Centro Atómico Ezeiza;

- Instituto Antártico Argentino (IAA). Con sede central en Miguelete (San Martín);

- Centro de Investigaciones Cientificas y Técnicas para la Defensa (CITEDEF). Tiene su sede central en Villa Martelli (Vicente López);
- Servicio Geológico Minero Argentino (SEGEMAR). Con sede central en Miguelete (San Martín);

- Administración Nacional de Laboratorios e Institutos de Salud (ANLIS). Cuenta con el Instituto Nacional de Enfermedades Virales Humanas ubicado en Pergamino y el Instituto Nacional de Epidemiología con sede en Mar del Plata;

- Comisión Nacional de Actividades Espaciales (CONAE). Con instalaciones en Punta Indio y Punta Alta;

- Servicio Meteorológico Nacional (SMN). Cuenta con estaciones de observación en diferentes puntos del territorio provincial.

Por último, en relación a las capacidades CyT instaladas en la provincia, es preciso destacar una diversidad de empresas de base tecnológica de carácter público y mixto (público/privado). En las mismas encontramos desde activos centros de I+D, hasta proyectos científico-tecnológicos de envergadura en sectores estratégicos como energía, salud, defensa, nuclear y satelital. Entre ellas podemos destacar: - YPF Tecnología (Y-TEC). Radicada en Berisso, dedicada a la industria energética;

- Nucleoeléctrica Argentina (NA-SA). Ubicada en el partido de Zárate, operadora de las centrales nucleares Atucha I y II, y a cargo de la gestión de proyectos nucleares como fue el CAREM;

- Empresa Argentina de Soluciones Satelitales (ARSAT). Con sede en Benavídez (Tigre), especializada en telecomunicaciones;

- Combustibles Nucleares Argentina (CONUAR). Ubicada en el Centro Atómico Ezeiza, orientada a combustibles nucleares, aleaciones especiales y otras manufacturas y servicios especiales;

Además del sector público, encontramos en el

\footnotetext{
${ }^{9}$ Elaboración propia a partir de datos obtenidos de CONICET en Cifras referidos al año 2018. Disponible en la página web del CONICET.
} 
sector privado un complejo entramado de empresas de base tecnológica. Por un lado, decenas de pequeñas y medianas empresas en sectores tales como software, química, petroquímica, energía, biotecnología, etc. Por lo general proveedoras de insumos y servicios tecnológicos a compañías mayores o bien, en el menor de los casos, comercializan e incluso exportan sus productos. Por otro lado, varias grandes empresas de origen nacional (Techint, Bagó, IMPSA, Roemmers, Pérez Companc, etc.) y algunas firmas transnacionales (3M, Oracle, IBM, etc.) con algún grado de investigación y desarrollo en el país. La presencia de este conjunto de empresas de base tecnológica explican por qué el promedio de inversión privada en I+D es mayor en la Provincia de Buenos Aires que en el resto del país.

\section{Capacidades CyT dependientes del Estado provincial}

Las políticas provinciales de CyT impulsadas desde la recuperación de la democracia en 1983 se han caracterizado por su escaso impacto en el aparato productivo y social de la provincia, sus magros presupuestos y su falta de continuidad. En una primera etapa, hasta fines de los '80, la ejecución de estas políticas se mantuvo a cargo de la Comisión de Investigaciones Científicas de la Provincia de Buenos Aires $(\mathrm{CIC})$, organismo descentralizado dependiente de la Gobernación. Pero, a partir de los años ‘90, se ejecutaron tales políticas, según cada gobierno, o bien desde subsecretarías dependientes de los Ministerios de Producción (1991-1998, 2002-2007, 2011-2015) o bien desde la Dirección General de Cultura y Educación (1999-2002, 2007-2011).

En 2016, durante la gobernación de María Eugenia Vidal, por primera vez se jerarquiza la cartera creando el Ministerio de Ciencia, Tecnología e Innovación.
Pero esta jerarquización sólo fue una estructura burocrática, al no estar acompañada de políticas y presupuesto adecuados, en un marco nacional de achicamiento y destrucción del sector caracterizado como un verdadero "cientificidio" (CLCPyS, 2016; Aliaga, 2019). Recientemente, el gobernador, Axel Kicillof, disolvió el Ministerio y volvió a crear una Subsecretaría de Ciencia y Tecnología, esta vez dentro del Ministerio de la Producción, Ciencia e Innovación Tecnológica. Esta constante inestabilidad institucional de la CyT en el organigrama provincial es expresión de la histórica ausencia de una política de Estado. En el mismo sentido, se destaca la falta de un plan estratégico provincial de ciencia y tecnología.

\section{La Comisión de Investigaciones Científicas de la Provincia de Buenos Aires}

En el marco de estas insuficiencias, el grueso de la actividad científico-tecnológica que ejecuta el Estado provincial se sigue concentrando en la CIC. Este organismo es una de las instituciones de СуT más antiguas del país, ya que fue creada en 1956 junto con el INTA, poco antes de la fundación del INTI (1957) y el CONICET (1958). Su función explícita es desarrollar investigación básica y aplicada que genere conocimiento e innovación y soluciones concretas al sector productivo provincial. Entre sus objetivos se destacan: formación de recursos humanos mediante becas de grado y posgrado, transferencia de conocimientos y tecnología a empresas y organismos de la provincia, servicios tecnológicos, certificaciones de productos y asesorías.

El personal de la CIC se compone de casi 900 personas, entre investigadores propios y asociados (156 + 80), profesionales y técnicos (199), becarios (320) y administrativos (130)..$^{10}$ Cuenta con carreras del investigador y personal de apoyo, y un sistema

\footnotetext{
${ }^{10}$ La información de personal refiere al año 2019 y se encuentra disponible en la página web de la CIC.
} 
de becas similares a las del CONICET. La edad promedio de sus investigadores y profesionales supera los cincuenta años y más del $70 \%$ son hombres, lo que da cuenta de una planta de personal envejecida y masculinizada. En cuanto a su infraestructura y despliegue, la CIC cuenta con 7 centros de I+D exclusivos del organismo, más 83 centros asociados con universidades nacionales instaladas en la provincia y otras instituciones como el CONICET.

Entre los principales problemas y desafíos que la $\mathrm{CIC}$ enfrenta actualmente se destacan:

Escasez presupuestaria. La falta de presupuesto ha sido una característica de las últimas décadas, pero se vio agravada durante la gestión de la gobernadora Vidal (2015-2019)." El deterioro presupuestario se expresa en magros salarios, falta de fondos para proyectos de I+D, infraestructura y equipamientos envejecidos, disminución en el número de becarios y escasos ingresos a las carreras de investigador y personal de apoyo (no logrando reponer siquiera la merma por jubilaciones y fallecimientos). Para dar cuenta de la gravedad de la situación, señalemos que, entre diciembre de 2015 y diciembre de 2019, la planta de investigadores propios cayó de 171 a 156, mientras que el personal de apoyo se redujo de 224 a 199.

Alto nivel de concentración geográfica. Casi la totalidad del personal, infraestructura y actividades de la CIC se concentran en la capital provincial, La Plata. De este modo, se desaprovecha una de las posibilidades más interesantes del organismo que es la inserción directa en los territorios.

Burocratización institucional. A pesar de ser un organismo descentralizado, la CIC depende excesivamente de la firma personal del gobernador. Esto tiene importantes consecuencias tales como una demora de años en trámites administrativos (ingresos y promociones, por ej.). En cuanto a la dinámica institucional, no sólo el Presidente de la CIC sino que todo el Directorio es designado directamente por el Poder Ejecutivo, sin mediar ningún tipo participación del personal del organismo. Por otra parte, hay una distorsión en la estructura de personal, ya que, por ejemplo, la proporción de investigadores/as en relación a los/as empleados/as administrativos/as es casi de paridad.

Ausencia de una política propia. La CIC se ha caracterizado por no tener una política institucional que oriente la actividad de su personal. En auto-evaluaciones realizadas en los últimos años en el organismo, aproximadamente el 80\% del personal manifestó desconocer cuáles son las directrices de la institución. En ese marco, se imponen políticas implícitas que conducen a una homologación con los criterios del CONICET en materia de evaluación, de promoción, de excelencia, etc.

\section{Otros organismos CyT provinciales}

En cuanto a otros organismos de CyT dependientes del Estado Provincial, cabe mencionar que existen instancias distribuidas en diversos ministerios que realizan algún tipo de actividad de I+D. Por ejemplo, en el ámbito del Ministerio de Salud se encuentra el Instituto Biológico Dr. Tomás Perón. Ubicado en la ciudad de La Plata, cuenta con una trayectoria relevante en el área de producción pública de medicamentos y productos biológicos. Además de la cartera de salud, se destacan dependencias de otros ministerios que realizan investigación aplicada (Economía, Educación, Producción, etc.). Por lo general se trata de investigaciones que sirven de insumo

\footnotetext{
${ }^{11}$ El presupuesto provincial en CyT para el año 2019 fue de $\$ 602.881 .430$, representando el $0.065 \%$ del presupuesto provincial total. De los cuales $\$ 481.573 .630$ corresponden a la CIC. Eso da un presupuesto mensual para la CIC (incluyendo salarios y becas para casi 900 personas) de apenas $\$ 40.131 .136$.
} 
para la toma de decisiones en la gestión pública.

En lo que respecta a la formación de recursos humanos, desde la descentralización educativa de los años '90 del siglo pasado, la Provincia de Buenos Aires cuenta con un importante entramado de instituciones de formación de nivel superior dependientes de la Dirección General de Cultura y Educación (DGCyE). En primer lugar, encontramos un total de 575 instituciones de formación docente y/o técnica de nivel terciario, de las cuales 299 son de gestión pública. La matrícula de estos institutos fue en 2019 de 268.000 estudiantes, con un $77 \%$ que asiste a instituciones de gestión pública. Cabe señalar que 118 de los institutos de gestión pública son de formación técnica (incluyendo anexos y extensiones).

Por otra parte, también en el nivel superior, la PBA cuenta con la Universidad Provincial de Ezeiza (UPE), inaugurada en 2011 y orientada a la actividad aeroportuaria, y la Universidad Provincial del Sudoeste (UPSO), puesta en marcha en el año 2000, con sede central en Pigüé y presencia en 26 localidades del sudoeste bonaerense. Entre las ofertas de formación técnica/tecnológica en otros niveles educativos, por un lado, la provincia cuenta con 454 escuelas técnicas profesionales, de las cuales 377 son establecimientos de gestión pública que reúnen 188.612 estudiantes (25.699 alumnos asisten a escuelas privadas).

Por otro lado, existen en la provincia 456 Centros de Formación Profesional (CFP), a los que asisten un total de 206.933 estudiantes. La oferta en este caso es extremadamente variada, abarcando desde formación en áreas técnicas específicas (Reparador de Equipos Electrónicos Industriales, Programador de Sistemas Informáticos, etc.) y oficios tradicionales (Gasista, Electricista, Soldador, etc.) hasta una infinidad de actividades laborales. ${ }^{12}$ Estas cifras dan una idea de la potencialidad que la provincia tiene para formar recursos humanos de nivel superior y/o técnico, que pueden ser utilizados en el fortalecimiento tanto del entramado productivo, como de las capacidades científico-tecnológicas.

\section{Propuestas}

Sobre la base del panorama analizado y de las capacidades con que cuenta la provincia, presentamos desde la Cátedra Libre Ciencia, Política y Sociedad de la Universidad Nacional de La Plata una serie de propuestas para que el sector de ciencia y tecnología pueda contribuir activamente al desarrollo provincial.

\section{Medidas de emergencia para enfrentar la crisis}

La grave situación socioeconómica por la que atraviesa la provincia después de cuatro años de neoliberalismo requiere poner a funcionar las capacidades científico-tecnológicas en función de resolver necesidades sociales y productivas urgentes. Desde la actual Subsecretaría de Ciencia y Tecnología se podría convocar a las Universidades y organismos de CyT ubicados en la provincia para la identificación de problemáticas y la generación de diagnósticos en sus respectivos territorios de influencia, que permitan elaborar agendas de I+D orientadas por demandas. Los focos iniciales más relevantes podrían estar puestos, por un lado, en las agendas de los municipios, y por otro lado, en los problemas y potencialidades de las cadenas de valor, con el objetivo de aumentar la integración y la productividad, en especial de las PyMEs y la producción popular. Siguiendo el modelo de la convocatoria nacional realizada en enero de 2020 por el MINCyT para colaborar con el Plan Argentina Contra el Hambre, podrían realizarse convocatorias específicas para

\footnotetext{
12 Todos los datos corresponden al año 2019 y están disponibles en DGCyE (2020).
} 
atender diferentes problemáticas de carácter urgente en materia de hábitat, salud, educación, trabajo, etc.

\section{Plan Estratégico Provincial de Ciencia y Tec- nología}

Contar con un Plan Estratégico Provincial de CyT (PPCyT) le daría coherencia a las políticas para el sector y facilitaría la articulación de las diferentes capacidades CyT con las que cuenta la provincia. Ayudaría, además, a orientar la formación de recursos humanos y la producción de conocimientos de acuerdo con los objetivos de desarrollo provincial. Un camino posible sería constituir, o consolidar en los casos en que ya existen, Consejos Locales y/o Regionales de Desarrollo, en los que participen, por un lado, PyMEs, cooperativas, empresas, sindicatos, movimientos sociales y de la economía popular, partidos políticos y sectores del Estado (vinculados entre otras con las áreas de producción, salud, educación, energía, hábitat y medio ambiente). Por otro lado, deberían participar actores vinculados directamente con la producción de conocimientos y con la formación de recursos humanos (CIC, institutos CyT, universidades, asociaciones profesionales, entre otros). La articulación intersectorial, interdisciplinaria y política de estos actores permitiría identificar y detallar las demandas económicas, sociales y ambientales de la provincia y realizar diagnósticos de necesidades. El resultado de este esfuerzo debería ser la elaboración de objetivos estratégicos, que se expresen en agendas de investigación que contemplen prioridades para el sector, selección de proyectos a desarrollar, orientaciones para la planificación educativa, generación de estudios prospectivos y criterios para formación de personal de las instituciones públicas. Para garantizar el cumplimiento y seguimiento de los objetivos y ajustar los planes, el PPCyT debería con- templar, además, diversas instancias de evaluación (observatorios, comisiones intersectoriales, instancias parlamentarias, etc.).

\section{Subsidios a proyectos de investigación orienta- dos por demandas}

Proponemos realizar convocatorias públicas para subsidiar proyectos estratégicos de investigación aplicada, destinados a brindar soluciones científico-tecnológicas inmediatas a problemas provinciales en áreas sensibles. Se trata de problemas en los que se define lo que se necesita y se convoca al sector CyT a proponer soluciones. La evaluación de estos proyectos no estaría guiada únicamente por la originalidad de la propuesta, sino además por su factibilidad y su impacto. La coordinación de estos proyectos podría ser tarea de la Subsecretaría de Ciencia y Tecnología, en interacción con la CIC, los ministerios y los municipios. Esta instancia establecería los criterios de evaluación y ejecución de acuerdo a los objetivos propuestos. En la selección se podría dar mayor relevancia a las propuestas que articulen instituciones y actores sociales y que integren grupos de investigación y equipos, a fin de optimizar capacidades y recursos. Dos antecedentes valiosos podrían servir de referencia para pensar estos nuevos proyectos. Por un lado, la experiencia de los Proyectos de Innovación y Transferencia en Áreas Prioritarias de la PBA (PIT-AP-BA) implementados por la CIC en 2016. Por el otro, las iniciativas impulsadas por el MINCyT y la ANPCyT frente a la pandemia de COVID19 en los meses de marzo y abril de 2020.

\section{Redefinición de la Comisión de Investigaciones Científicas (CIC)}

La CIC debería tener una mayor jerarquía para poder jugar un rol más activo y relevante en la ejecución de las políticas CyT provinciales. La institución requiere 
un rediseño institucional y un mayor presupuesto a fin de lograr una mejor articulación con los sectores productivos y sociales de la provincia y un más ágil y efectivo funcionamiento. Creemos que no se trata de tener un "pequeño CONICET", sino una "gran CIC" volcada a las problemáticas bonaerenses. Los centros de investigación propios deberían tener políticas institucionales de acuerdo al Plan Provincial de Ciencia y Tecnología. Consistentemente, se debería redefinir el perfil de los investigadores y becarios mediante una revisión de las pautas de evaluación, para que sean coherentes con los objetivos propuestos. Acompañando estos cambios, es preciso impulsar una democratización del organismo, incorporando representantes de los distintos estamentos en el Directorio, elegidos mediante el voto directo. De este modo se lograría una mayor identificación con los lineamientos que emanan de la dirección del organismo. Por otro lado, se debería transformar el sistema de becas doctorales y postdoctorales en contratos a término, con plenos derechos laborales. Se requiere además revisar y reformar la Ley del Investigador de la Pcia. de Bs.As. También, impulsar políticas que tiendan a una mayor equidad de género, promoviendo mecanismos para que haya más ingresos de trabajadoras.

\section{Conformación de un Polo Científico-Tecnológico}

En el marco de la redefinición de la CIC se podría impulsar la conformación de un Polo Científico-Tecnológico en el Campus que la misma posee en Gonnet (La Plata). Con carácter multidisciplinario, articularía soluciones tecnológicas con demandas económicas, sociales y ambientales de la provincia. Incluiría a los 7 centros de la $\mathrm{ClC}$, que actualmente allí se encuentran, de los cuales 4 son exclusivos del organismo y 3 son de múltiple dependencia con diferentes organismos nacionales (CONICET, UNLP, INTI). Podría estar integrado además por otros institutos de la $\mathrm{ClC}$, que sin ser parte del Campus aportarían sus capacidades para la construcción de este Polo. Además, sería posible incorporar empresas de base tecnológica. Podría estar compuesto por un Área de Servicios Tecnológicos a la Industria, orientado sobre todo a las PyMEs, un Área de Desarrollo Tecnológico e Innovación, dedicada al diseño de productos y la mejora de métodos y técnicas, un Área de Investigación Estratégica, orientada a demandas de conocimientos por parte del Estado en áreas sensibles como energía, infraestructura, comunicaciones, etc., y un Área de Tecnologías Sociales, dedicada a las problemáticas tecnológicas de la producción popular. Experiencias a tener en cuenta en la construcción de este Polo Científico-Tecnológico son la Fundación Tecnología Electrónica de Alta Complejidad (TEAC), ubicada en Bahía Blanca y constituida por el INTI y nueve empresas tecnológicas, los clusters vinculados al sector de software instalado en la provincia, y los programas analizados por Briozzo (2011) desarrollados en el período 2008-2010 por la CIC.

\section{Transferencia de tecnología hacia la producción popular}

La provincia de Buenos Aires tiene una importante actividad económica informal en la que se desenvuelven millones de trabajadores, denominada economía popular. En muchos casos se trata de cooperativas, dedicadas a labores con baja complejidad tecnológica en rubros como producción de alimentos, construcción y reparación de viviendas, recuperación de residuos urbanos, manufactura textil, etc. Desde el sector CyT podrían brindarse soluciones técnicas, o complementar respuestas socio-técnicas en curso, para estas actividades, permitiendo a estos sectores el desarrollo y adopción de las tecnologías convenientes. En particular, se podrían mejorar las condiciones de trabajo y permitir la apropiación de una mayor parte de la renta económica mediante el agregado de valor. En otras palabras, proponemos impulsar el desarrollo de tecnología 
| Ciencia, Tecnología y Política | Año 3 | No4 | Mayo 2020 | ISSN 2618-3188 | www.revistas.unlp.edu.ar/CTyP |

para la producción popular, mediante la dupla economía solidaria-tecnología social (Martínez, 2017). Por ejemplo, brindando acceso a tecnologías para gestión y comercialización, automatización de procesos sencillos, manejo de fuentes alternativas de energía, reciclado, tratamiento de residuos y efluentes, etc. Podría articularse con la educación técnica secundaria y terciaria y las universidades, instituciones que tienen un gran despliegue territorial. Se debería, además, impulsar el Proyecto de Ley de Promoción de Transferencia de Tecnología a la Producción Popular presentado en el Congreso Nacional (IPP, 2016).

\section{Utilizar el poder de compra del Estado para dinamizar las empresas de base tecnológica}

El poder de compra del Estado es una poderosa herramienta para apuntalar la producción local de tecnología. Por ello, proponemos impulsar el uso de las llamadas compras públicas de tecnología, un procedimiento habitual a nivel nacional y en diversos países. Éstas se realizan cuando una entidad pública aprueba el pedido de un producto o sistema que no existe en ese momento y que se requiere para el desarrollo de una tecnología nueva, o para mejorar una ya existente. Esta opción de financiamiento permite apoyar soluciones tecnológicas para áreas estratégicas. La novedad de este mecanismo de compra estaría en que la licitación se realiza a través de la definición de funciones y objetivos, y no de productos conocidos. En otras palabras, se sabe lo que se necesita, pero no cómo se lo va a resolver. Esta política ayudaría a que la industria nacional y especialmente las PyMEs desarrollen autonomía tecnológica y se creen consorcios entre instituciones públicas y empresas (públicas, mixtas o privadas), de modo de promover la sinergia entre actores productivos y el sector CyT.

\section{Aumento del presupuesto provincial para el sec- tor de CyT}

Generar una política CyT que pueda contribuir a re- solver los problemas y necesidades de la provincia requiere aumentar la participación de esta función en el presupuesto provincial. Como medida urgente, es preciso aumentar los salarios de investigadores/ técnicos/administrativos de la CIC y el monto de las becas para recuperar el deterioro de los ingresos sufrido en los últimos años. Más allá de estas urgencias, es preciso poder contar con una Ley Provincial de Financiamiento a la Ciencia, la Tecnología y la Innovación. La ley debería contemplar, por un lado, el rol del complejo científico-tecnológico provincial como consultor estratégico del Estado. Por otro lado, podría destinar fondos para promover convenios con municipios interesados en radicar en sus distritos empresas de base tecnológica, o centros de investigación y/o educación superior, y proyectos generados por comunidades locales y regionales. Asimismo, destinar recursos que estimulen la radicación de investigadores en el interior de la provincia. Dado que el erario público está seriamente comprometido, la ley debería contemplar diversas alternativas y estrategias de financiamiento (alícuotas diferenciales, exenciones impositivas como fomento a la inversión privada en I+D, Programas de crédito fiscal,etc.).

\section{Bibliografía}

Aliaga, J. (2019). Ciencia y tecnología en la Argentina 2015-2019. Ciencia, tecnología y política, 2(3), 024. https://doi.org/10.24215/26183188e024

Basualdo, E., Manzanelli, P. \& Bona, L. (2019). Estructura económica y desintegración productiva en tiempos recientes. En Kicillof, A. et al. (2019). Radiografía de la provincia de Buenos Aires. Buenos Aires: Siglo $X X \mid$.

Briozzo, A. N. (noviembre, 2011). Promoción de la innovación e incorporación de soluciones tecnológicas en micro, pequeñas y medianas empresas: análisis de la metodología y resultados (2008-2010) del Programa de Modernización Tecnológica (PMT) 
de la Comisión de Investigaciones Científicas de la Provincia de Buenos Aires, Argentina. Ponencia presentada en 9th GLOBELICS International Conference, Buenos Aires, Argentina.

CLCPyS (2016). Ajuste, achicamiento y cientificidio: la ciencia en el primer año de gobierno de Mauricio Macri. Documento de la Cátedra Libre Ciencia, Política y Sociedad de la UNLP publicado en diciembre de 2016. Recuperado de: http://sedici.unlp.edu.ar/ handle/10915/62256

Legislatura de la Provincia de Buenos Aires (2019). Presupuesto. Ley 15.078. Recuperado de https://n9. $\mathrm{cl} / \mathrm{vhml}$

DGCyE (2020). Estadística Educativa Anual 2019. La Plata: Gobierno de la Provincia de Buenos Aires Recuperado de: https://n9.cl/1s1gt (última visita el 16 de abril de 2020).

IPP (29 de junio de 2016). Proyecto de Ley de Promoción de Transferencia de Tecnología a la Producción Popular. Instituto para la Producción Popular. Recuperado de https://n9.cl/7td3

Martínez, E. (2017). Ocupémonos: del Estado de Bienestar al Estado Transformador. Edición de autor. Ministerio de Hacienda y Finanzas (2020). Informe de deuda pública de la Provincia de Buenos Aires: estado de situación a diciembre de 2019. La Plata: Gobierno de la Provincia de Buenos Aires. Recuperado de: https://n9.cl/g3rO (última visita el 16 de abril de 2020).

OCTS (2018). Las universidades, pilares de la ciencia y la tecnología en América Latina. Contribución del Observatorio Iberoamericano de la Ciencia, la Tecnología y la Sociedad de la OEl a la III Conferencia Regional de Educación Superior (CRES2018), Junio
2018, Córdoba, Argentina. Recuperado de: https:// n9.cl/Ok01

SPU (2019). Sistema de consulta de estadísticas universitarias. Disponible en Secretaría de Políticas Universitarias https://n9.cl/pam9 [Fecha de consulta: 26-2-2020]. 